$10 ; 11 ; 15$

\title{
Магнитоакустический генератор хаотических колебаний с задержкой и бистабильностью
}

\author{
(C) А.Н. Литвиненко ${ }^{1}$, С.В. Гришин ${ }^{1}$, Ю.П. Шараевский ${ }^{1}$, \\ B.В. Тихонов ${ }^{1}$, С.А. Никитов ${ }^{1,2}$ \\ ${ }^{1}$ Саратовский государственный университет им. Н.Г. Чернышевского, \\ Саратов, Россия \\ ${ }^{2}$ Институт радиотехники и электроники им. В.А. Котельникова, Москва, \\ Россия \\ E-mail: litvinenkoan@gmail.com
}

Поступило в Редакцию 18 мая 2017 г.

Приводятся экспериментальные результаты формирования хаотических колебаний в неавтономном магнитоакустическом генераторе, выполненном на основе гибридного магнитоакустического резонатора отражательного типа. Указанный генератор под внешним низкочастотным периодическим воздействием демонстрирует режимы квазипериодической и хаотической генерации. Хаотическая динамика магнитоакустического генератора обусловлена наличием в системе задержки и бистабильности.

DOI: 10.21883/PJTF.2018.06.45771.16880

Хаотические генераторы микроволнового диапазона находят широкое применение в системах скрытой передачи информации и хаотической радиолокации $[1,2]$. Их чувствительность к начальным условиям и параметрам динамической системы позволяет осуществлять секретную передачу данных на хаотической несущей. В последние годы в качестве нелинейных элементов в генераторах хаоса стали использоваться пленки железо-иттриевого граната (ЖИГ), в которых трех- и четырехволновые процессы взаимодействия спиновых волн являются причиной генерации хаотического сигнала [3-5]. Другая возможность создания хаотических генераторов - использование деформаций, возникающих в результате магнитоупругого взаимодействия магнитостатических спиновых волн (МСB) со звуковыми волнами. Исследования магнитоупругого взаимодействия и разработка на его основе различных устройств 
функциональной электроники активно ведутся в последние десятилетия $[6,7]$. В рамках этих исследований в [8] был проведен анализ волновых процессов в гибридном магнитоакустическом резонаторе (MAР), представляющем собой прямоугольный резонатор на основе пленки ЖИГ, выращенной на подложке гадолиний-галлиевого гранта (ГГГ), с плоскопараллельными поверхностями. В [9] было установлено, что такой резонатор, имея высокую собственную добротность $Q_{0} \approx 5000$, способен дискретно перестраиваться магнитным полем в широкой полосе частот и может использоваться для создания высокостабильных задающих генераторов с низким уровнем фазовых шумов. Кроме того, как показали предварительные экспериментальные исследования, разработанный магнитоакустический резонатор при своих геометрических размерах обладает двумя характерными временами: временем задержки сигнала в акустической подложке ГГГ $\tau_{d e l}=0.2078 \mu \mathrm{s}$, а также временем установления в ней акустических колебаний $\tau_{s e t} \approx 3 \mu \mathrm{s}$. Первое из характерных времен определяет частотный интервал между акустическими резонансами, а второе - добротность акустического резонанса, образующегося на резонансной кривой МСВ-резонатора. Наличие такого резонанса приводит к появлению двух устойчивых состояний у автоколебательной системы, выполненной на основе магнитоакустического резонатора, и как следствие этого к возникновению в системе бистабильности. Для получения режимов динамического хаоса в динамических системах используются механизмы как задержки [10], так и бистабильности [11].

В настоящей работе представлены экспериментальные результаты исследования хаотической динамики автогенератора на основе магнитоакустического резонатора, который находится под внешним периодическим параметрическим воздействием.

На рис. 1 приведена блок-схема магнитоакустического генератора (МАГ), разработанного в классической структурной схеме на отражение с отрицательным сопротивлением. Генератор собран на основе микросхемы D1 MAX2620, которая содержит активные элементы, цепи обратной связи и буферные усилители и действует как отрицательное сопротивление в области частот от $500 \mathrm{MHz}$ до $1 \mathrm{GHz}$. Между полюсами электромагнита, создающего постоянное магнитное поле $H_{0}$, помещается проволочная микрокатушка с индуктивностью $L=10 \mu \mathrm{H}$, к которой прикладывается напряжение $U_{1}=U_{0} \cos \left(2 \pi f_{\text {ext } t} t\right)\left(U_{0}-\right.$ амплитуда напряжения, $f_{\text {ext }}-$ частота внешнего воздействия) для 


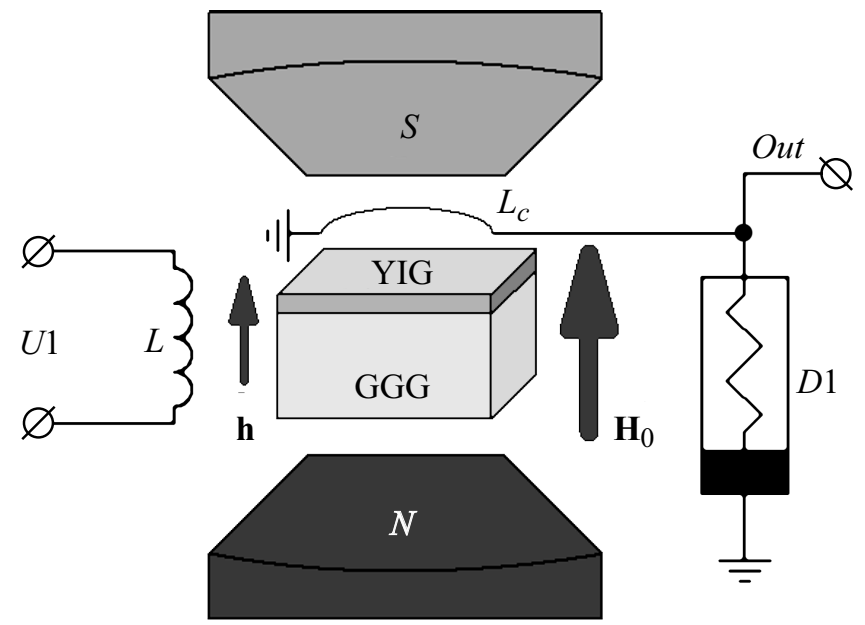

Pис. 1. Блок-схема магнитоакустического генератора с магнитоакустическим резонатором.

создания переменного подмагничивающего поля. В этом случае на MAР действует эффективное магнитное поле $H(t)=H_{0}+h(t)$, где $h(t)=h_{0} \cos \left(2 \pi f_{e x t} t\right)$, амплитуда переменной компоненты которого $h$ пропорциональна амплитуде напряжения $U_{0}$.

Магнитоакустический резонатор изготовлен на основе пленки ЖИГ толщиной $d=13 \mu \mathrm{m} \mathrm{c}$ намагниченностью насыщения $4 \pi M_{0}=1740 \mathrm{G}$, выращенной методом жидкофазной эпитаксии на подложке ГГГ толщиной $D=358 \mu \mathrm{m}$. Внешнее постоянное магнитное поле $H_{0}=2060 \mathrm{Oе}$ прикладывается нормально к поверхности МАР, который характеризуется латеральными размерами $1 \times 1 \mathrm{~mm}$. В качестве рабочей моды выступает первая неоднородная мода прямой объемной МСВ (ПОМСВ) с волновым числом $k=5 \mathrm{~cm}^{-1}$, для которого удовлетворяются резонансные условия в пленке ЖИГ. Для возбуждения ПОМСВ в пленке ЖИГ используется индуктивный преобразователь, выполненный в виде проволоки из меди толщиной $0.2 \mathrm{~mm}$ и индуктивностью $L_{c}=5 \mathrm{nH}$. Преобразователь расположен на расстоянии $0.1 \mathrm{~mm}$ от поверхности пленки ЖИГ. Поверхности МАР отполированы и доведены до плоскопараллельного состояния для уменьшения акустических потерь,

Письма в ЖТФ, 2018, том 44, вып. 6 


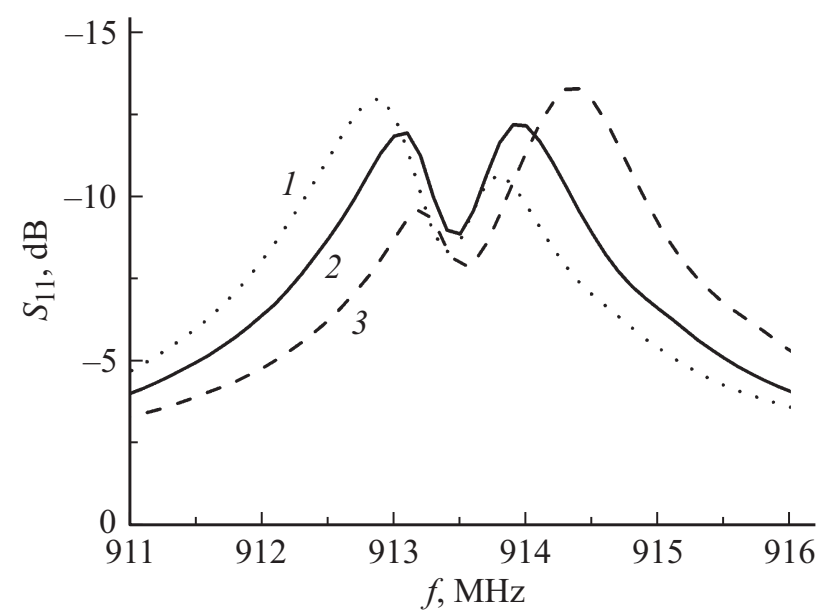

Рис. 2. Амплитудно-частотные характеристики МАР, полученные при различных значениях эффективного магнитного поля. $H_{0}$, Oe: $1-319.1,2-318.9$, $3-319.4$.

возникающих у объемной сдвиговой звуковой волны, возбуждаемой ПОМСВ в подложке ГГГ через магнитоупругое взаимодействие. Угол клина между гранями МАР составляет менее 2 arcsec.

На рис. 2 (кривая 2) приведена частотная зависимость модуля коэффициента отражения разработанного МАР, которая отличается от амплитудно-частотной характеристики (АЧХ) традиционного прямоугольного МСВ-резонатора [12]. В исследуемом МАР из-за возбуждения звуковой волны в подложке ГГГ на фоне резонансного пика МСВ-резонатора появляется высокодобротный резонансный пик, направленный в обратную сторону и связанный с перекачкой энергии МСВ в резонирующую в подложке ГГГ звуковую волну.

При подключении МАР к схеме с отрицательным сопротивлением (рис. 1) и подаче постоянного напряжения $\left(f_{\text {ext }}=0\right)$ на катушку индуктивности $L$ возникает генерация монохроматического сигнала на частоте одного из двух пиков АЧХ МАР в зависимости от случайных флуктуаций при включении генератора (рис. 2, кривая 2). Изменение постоянного напряжения $U_{0}$ приводит к изменению величины эффективного магнитного поля и как следствие этого к сдвигу частоты МСB-

Письма в ЖТФ, 2018, том 44, вып. 6 
резонатора. АЧХ МАР становится несимметричной (рис. 2 , кривые 1,3 ). При этом сигнал может генерироваться в том числе на частоте, соответствующей меньшему по величине пику АЧХ МАР, до тех пор, пока его относительная амплитуда не станет меньше амплитуды второго (большего по величине) пика на $5 \mathrm{~dB}$. При превышении этого порогового значения происходит перескок частоты генерации на частоту, соответствующую большему по величине пику АЧХ МАР. При изменении величины эффективного магнитного поля в прямом и обратном направлениях частота генерации имеет разные зависимости от поля, что указывает на присутствие в автоколебательной системе бистабильности и гистерезиса.

На рис. 3 представлены результаты экспериментального исследования режимов генерации МАГ при подаче переменного напряжения на проволочную микрокатушку. В автономном режиме в динамической системе формируется монохроматический сигнал на частоте $f_{0}=913.5 \mathrm{MHz}$, которому в фазовом пространстве мгновенная частота сигнала-значение переменного подмагничивающего поля соответствует неподвижная точка. Анализ частоты МАГ проводился с использованием преобразования Гильберта, которое позволяет отслеживать изменение во времени фазы сигнала и его мгновенной частоты. В неавтономном режиме при подаче на проволочную микрокатушку переменного напряжения с частотой $f_{\text {ext }}=300 \mathrm{kHz}$ и амплитудой $U_{0}=320 \mathrm{mV}$ переменное подмагничивающее поле имеет амплитуду $h_{0}=68 \mathrm{mOe}$. В спектре сигнала (рис. $3, a$ ) появляются модуляционные составляющие с частотами $f_{0} \pm n f_{\text {ext }}$, где $n=1,2,3, \ldots$, являющиеся результатом частотной модуляции сигнала МАГ. Значение частоты $f_{\text {ext }}$ выбиралось таким образом, чтобы оно соответствовало времени установления акустических колебаний $\tau_{s e t}$ в подложке ГГГ. Исходя из анализа экспериментальных данных установлено, что изменение мгновенной частоты генератора происходит в интервале частот, находящемся вблизи частоты одного из пиков МАР. В фазовом пространстве наблюдается предельный цикл (рис. $3, b$ ).

Увеличение амплитуды переменного подмагничивающего поля до $h_{0}<110 \mathrm{mOe}$ приводит к увеличению количества модуляционных составляющих и их амплитуды что обусловлено нелинейностью усилительного каскада генератора. В фазовом пространстве по-прежнему наблюдается предельный цикл. При амплитуде переменного подмагничивающего поля $h_{0}=110 \mathrm{mOe}$ (рис. $3, c$ ) интервал изменения

Письма в ЖТФ, 2018, том 44, вып. 6 

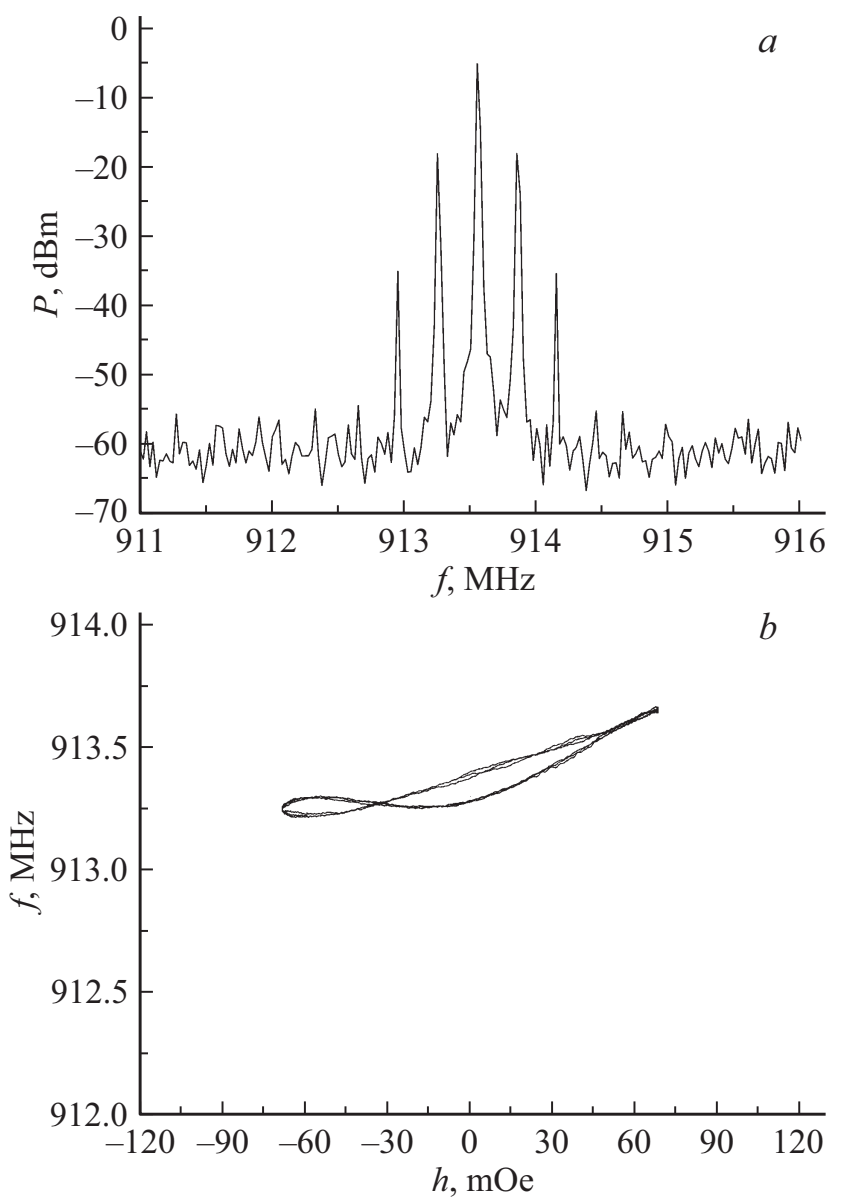

Рис. 3. Спектры мощности $(a, c)$ и фазовые портреты $(b, d)$ сигнала, генерируемого МАГ при двух значениях амплитуды $h_{0}$ переменного магнитного поля: $68(a, b)$ и $110 \mathrm{mOe}(c, d)$.

мгновенной частоты ПОМСВ-резонатора становится приблизительно равным ширине области гистерезиса, что приводит к хаотическому переключению частоты генерации между двумя пиками АЧХ МАР и

Письма в ЖТФ, 2018, том 44, вып. 6 

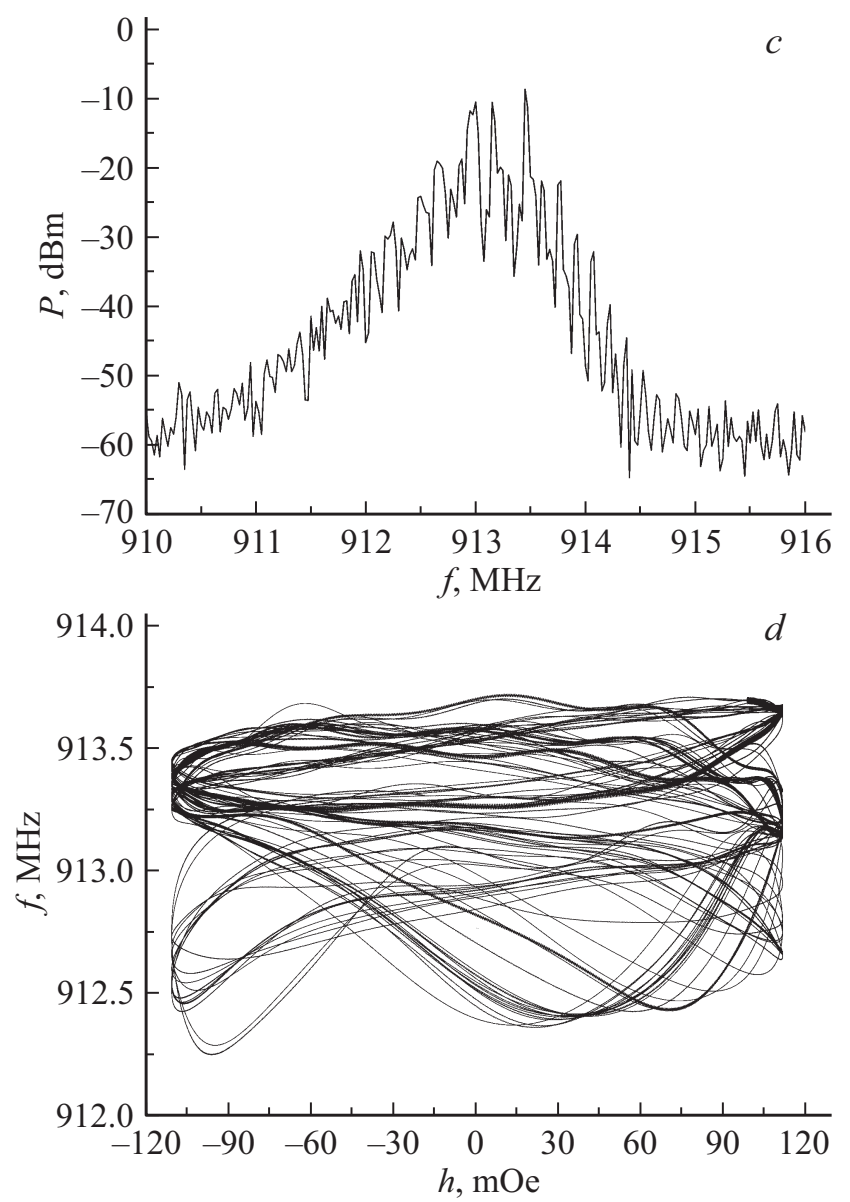

Рис. 3 (продолжение).

как следствие этого к образованию сплошного спектра у сигнала. Хаотическое переключение частоты генерации становится возможным тогда, когда на АЧХ МАР акустический резонанс не успевает сформироваться полностью за время $\tau_{s e t}$. В этом случае отсутствует детерминированный переход между интервалами генерации на правом и левом пиках

Письма в ЖТФ, 2018, том 44, вып. 6 
АЧХ МАР. В момент максимального отклонения резонансной частоты ПОМСВ-резонатора вероятность перехода точки генерации на другой пик АЧХ МАР определяется амплитудой и частотой задержанного в акустической подложке сигнала, т.е. тем насколько в ней успел сформироваться акустический резонанс. Фазовый портрет, соответствующий данному режиму генерации, напоминает по виду аттрактор типа double scroll (рис. $3, d$ ), который свойствен фазовым пространствам с двумя бассейнами притяжения и неустойчивой седлоузловой точкой посередине.

В заключение отметим, что в отличие от известных сегодня генераторов хаоса на основе МСВ генератор, разработанный на базе магнитоакустического резонатора, демонстрирует исключительно фазочастотный хаос, который может быть использован для создания источников фазоманипулированных сигналов для систем скрытой передачи информации.

Авторы выражают благодарность Р.С. Химину, В.С. Тиберкевичу и А.Н. Славину за проявленное внимание к работе и плодотворное обсуждение полученных результатов.

Работа выполнена при финансовой поддержке РФФИ (грант № 1629-14021-офи-м).

\section{Список литературы}

[1] Halle K.S., Wu C.W., Itoh M., Chua L.O. // Int. J. Bifurcation Chaos. 1993. V. 3. N 2. P. $469-477$.

[2] Dmitriev A.S., Kyarginsky B.Ye., Panas A.I., Starkov S.O. // Int. J. Bifurcation Chaos. 2003. V. 13. N 6. P. 1495-1507.

[3] Демидов В.Е., Ковииков Н.Г. // Письма в ЖТФ. 1998. Т. 24. В. 7. С. 66-72.

[4] Гришин С.В., Гришин В.С., Храмов А.Е., Шараевский Ю.П. // ЖТФ. 2008. T. 78. B. 5. C. $89-98$.

[5] Кондрашов А.В., Устинов А.Б., Калиникос Б.А. // Письма в ЖТФ. 2016. T. 42. B. 4. C. $82-89$.

[6] Гуляев Ю.В., Зильберман П.Е., Казаков Г.Т., Сысоев В.Г., Тихонов В.В., Филимонов Ю.А., Нам Б.П., Хе А.С. // Письма в ЖЭТФ. 1981. Т. 39. В. 9. C. 500-504.

[7] Казаков Г.Т., Тихонов В.В., Зильберман П.Е. // ФТТ. 1983. Т. 25. В. 8. C. $2307-2311$. 
[8] Litvinenko A.N., Sadovnikov A.V., Tikhonov V.V., Nikitov S.A. // IEEE Magn. Lett. 2015. V. 6. P. 99-104.

[9] Тихонов В.В., Литвиненко А.Н. // Материалы XVII Междунар. зимней школы-семинара по электронике сверхвысоких частот и радиофизике: Саратов, 2015. С. 61.

[10] Loiko N.A., Samson A.M. // Quantum Electron. 2007. V. 24. N 8. P. 657-661.

[11] Shekhawat A., Kalmar-Nagy T. // Physica D. 2009. V. 238. N 17. P. 1768-1786.

[12] Marcelli R., Gasperis P.D. // IEEE Trans. Magn. 1991. V. 27. N 6. P. 5477-5479.

Письма в ЖТФ, 2018, том 44, вып. 6 